\title{
A Panel of Circulating Non-coding RNAs in the Diagnosis and Monitoring of Therapy in Egyptian Patients with Breast Cancer
}

Nadine Wehida ( $\sim$ nadinewehida89@gmail.com )

Medical Research Institute, Alexandria University

Wafaa Abdel-Rehim

Medical Research Institute, Alexandria University

Hazem El Mansy

Medical Research Institute, Alexandria University

Ahmed Karmouty

Medical Research Institute, Alexandria University

Maher A. Kamel

Medical Research Institute, Alexandria University

\section{Research Article}

Keywords: Breast, cancer, diagnostic, non-coding RNA

Posted Date: February 28th, 2022

DOI: https://doi.org/10.21203/rs.3.rs-1388989/v1

License: (c) (1) This work is licensed under a Creative Commons Attribution 4.0 International License. Read Full License 


\section{Abstract}

1) Background: Non coding RNAs (ncRNAs) have recently been identified to play a pivotal role in many diseases including breast cancer (BC). This study aims to investigate the relative quantification of long non-coding RNA (IncRNA) H19, microRNA (miR) 675-5p, 675-3p and miR-let 7 in BC patients.

2) Methods: The study was performed on three groups, group $1 ; 30$ non-intervened $B C$ female patients about to undergo breast surgery, group 2; 30 postoperative female BC patients about to receive adjuvant anthracycline chemotherapy and group 3; 30 apparently healthy female volunteers as the control group. Plasma samples were drawn before and after the intervention in groups 1 and 2 , with a single sample drawn from group 3. The relative quantification levels were compared with healthy control subjects and were related with the clinicopathological statuses of these patients.

3) Results: There was a statistically significant increase in H19, miR-675-5p, miR-675-3p and miR-let 7 in the non-intervened BC patients when compared to the control group. Surgery resulted in a significant reduction in all four ncRNAs under investigation. Chemotherapy resulted in a significant increase in the level of miR-let 7. The assay discriminated normal from $\mathrm{BC}$ where the area under the receiver operating characteristic curve (AUC-ROC) of miR-675-3p showed the maximal AUC-ROC of 1.000. The diagnostic sensitivity and specificity was also $100 \%$ when CA $15-3$ and $\mathrm{H} 19$ were combined.

4) Conclusion: The results indicate that the panel of ncRNAs can all potentially act as novel biomarkers whether alone or combined in the diagnosis of BC.

\section{Introduction}

NcRNAs constitute $99 \%$ of the gross cellular RNA [1] and are classified broadly into housekeeping and regulatory ncRNAs. Ribosomal RNA (rRNA), transfer RNA (tRNA), small nuclear RNA (snRNA) and small nucleolar RNA (snoRNA) constitute the housekeeping RNAs. Regulatory RNAs are further classified into long ncRNAs ( $>200$ nucleotides) and short ncRNAs ( $<200$ nucleotides) [2].

Findings of research studies on the diagnostic power of IncRNA are still conflicting and controversial. In this article, a panel of four ncRNAs will be assessed in BC patients. LncRNA H19; a member of the IncRNA family, is a paternally imprinting gene located in chromosome 11p15.5. It is a potential marker in cancer detection which has been postulated to be useful in therapy signifying chemosensitivity and drug resistance. [4] Both variants of its descendant miR-675, which was originally demonstrated to restrict the growth of the placenta prior to birth [6]; $5 p$ and 3p, which have been implicated in cell viability, invasion and mestastasis, making them a potential target in BC treatment. [7] As well as let 7, one of the first miRNAs discovered in Caenorhabditis elegans, which has been linked to chemoresistance and inhibition of the growth of $\mathrm{BC}$ cells upon downregulation. [3, 8]

Several oncogenic pathways are involved in $\mathrm{BC}$ invasion and progression, therefore the use of functional molecules can serve as potential novel markers in tumor onset and progression. Currently used 
diagnostic biomarkers such as cancer antigen 15 - 3 (CA 15 - 3) and carcinoembryonic antigen (CEA), have been used skeptically as markers in cancer diagnosis due to their poor reliability in identifying cancer cases accurately, this in turn has made the quest of alternative diagnostic biomarkers with more reproducible and accurate results a research pursuit. [9]. The identification of valid conclusive biomarkers in the detection of $\mathrm{BC}$ and other types of cancers could replace the invasiveness of unnecessary pathology biopsies and the downsides of mammography.

\section{Materials And Methods}

2.1. Sample collection: After approval of the Ethical Committee of the Medical Research Institute, Alexandria University, ninety female subjects were included in this study. Sample collection was carried out from October 2019 to January 2021. All patients were recruited from the Surgery and Cancer Management and Research departments. Plasma samples were drawn from three groups each comprising of 30 subjects; non-intervened $\mathrm{BC}$ patients, $\mathrm{BC}$ paients about to start adjuvant anthracycline chemotherapy and a control group. Two samples were drawn from each patient; a week before surgery and a week after surgery from the surgery group and two days before the first session and a week after the final session of the anthracyline adjuvant chemotherapy treatment from the chemotherapy group. A single sample was drawn from a third control group of 30 healthy female volunteers. Patients with diabetes mellitus, coronary artery disease, liver disease, asthma, sickle cell anemia, thyroid disease as well pregnant women were excluded.

To all the subjects in the study a full clinical evaluation and pathological investigation were recorded.

2.2. Sample preparation: Five milliliters of blood were collected, $3 \mathrm{ml}$ of which in ethylene diamine tetraacetic acid (EDTA) tubes, then centrifuged for 10 minutes at $6000 \mathrm{rpm}$ to collect plasma, $2 \mathrm{ml}$ were left to coagulate for 30 minutes then centrifuged for 10 minutes at $3000 \mathrm{rpm}$ to collect the serum. The collected plasma was used for the complete blood count (CBC) analysis and for the assay of the ncRNAs in question. The serum was divided into two aliquots, one for the CA $15-3$ analysis and the second for the routine biochemical tests. The routine biochemical tests and CA $15-3$ were analyzed on the same day. The plasma samples were encrypted and stored at $-80^{\circ} \mathrm{C}$ till the time of analysis.

2.3. Laboratory investigation: Complete blood picture and morphological examination, serum glucose, creatinine, alkaline phosphatase (ALP) activity, alanine aminotransferase (ALT) activity, aspartate aminotransferase (AST) activity and CA $15-3$. Laboratory investigations were performed to identify comorbidities and subjects for exclusion.

2.4. RNA extraction: Total RNA was isolated from the plasma samples using miRNeasy Mini Kit (Qiagen, Germany, catalog number 217061) according to the manufacturer instructions. The miRNeasy Mini Kit combines phenol/guanidine-based lysis of samples and silicon membrane - based purification of total RNA. QIAzol Lysis Reagent, included in the kit, is a monophasic solution of phenol and guanidine thiocyanate, designed to facilitate lysis of tissues, to inhibit RNases and also to remove most of the cellular DNA and proteins from the lysate by organic extraction. 
2.5. Reverse transcription: Reverse transcription was done using miScript II RT Kit (Qiagen, Germany, catalog number 218161) according to the manufacturer instructions. The miScript II RT Kit was used to perform a one-step, single-tube reverse transcription reaction. miScript HiFlex Buffer promotes conversion of all RNA species (mature miRNA, precursor miRNA, noncoding RNA, and mRNA) into cDNA. This enables flexibility to study miRNA biogenesis and genome wide miRNA and mRNA regulation in a single cDNA sample.

2.6. Relative quantification: Quantitative PCR (qPCR) was applied to determine the circulating levels of IncRNA H19, miR-675-5p, miR-675-3p and miR-let 7 using Rotor-Gene Q-Pure Detection version 2.1.0 (build 9) (Qiagen, USA), with cycling conditions of an initial activation of 10 minutes at $95^{\circ} \mathrm{C}, 40$ cycles, 15 seconds each; a denaturation at $94^{\circ} \mathrm{C}$, annealing at $55^{\circ} \mathrm{C}$ and extension at $60^{\circ} \mathrm{C}$ when data acquisition was collected. Real-time PCR quantification of mature miRNA and ncRNA was done using target-specific miScript Primer Assays (forward primers) and the miScript SYBR Green PCR Kit (catalog number 218073), which contains the miScript Universal Primer (reverse primer) and QuantiTect SYBR Green PCR Master Mix. The relative quantification (RQ) using comparative threshold cycle (Ct) provides an accurate comparison between the initial levels of template in each sample. The key feature of real-time PCR is that the amplified DNA is detected as the reaction progresses in real time and data is collected throughout the PCR process. A normalizer or reference gene (miRNA-16-5p) was used as an internal control for experimental variability in this type of quantification. This method of relative quantification is proposed by Livak et al. [10]

\section{Results}

\subsection{Statistical analysis}

CA 15 - 3 serum levels were significantly higher in the two groups of BC patients; who underwent surgery only or adjuvant chemotherapy (both pre and post treatment) compared with the control group. CA $15-3$ dropped significantly after surgery yet did not show a significant change after chemotherapy (Fig. 1).

The circulating $\mathrm{H} 19$ levels were significantly higher in the BC patients of the surgery group (pre and postsurgery) compared with the control group. The post-surgery level showed significant decline compared with the pre-surgery level. Regarding the patients of chemotherapy group, the prechemotherapy levels of $\mathrm{H} 19$ are comparable with the control levels and significantly lower than the post-surgery level. The postchemotherapy levels of circulating H19 are significantly lower than the control group (Fig. 2).

The circulating miR-675-5p levels were significantly higher in the BC patients pre and post-surgery compared with the control group. The post-surgery level showed significant decline compared with the pre-surgery level. Regarding the patients of chemotherapy group, the prechemotherapy levels of miR-675$5 p$ are not differ from the control levels, however, significantly lower than the post-surgery levels. The post-chemotherapy levels of circulating miR-675-5p are significantly higher than the control group (Fig. $3)$. 
The circulating levels of miR-675-3p showed significantly higher levels in all the BC patients; pre and postsurgery and pre and post-chemotherapy, compared with the control group. The highest circulating levels of miR-675-3p observed in the pre-surgery patients and the lowest levels were detected in the postchemotherapy patients. The post-surgery levels were significantly lower than the pre-surgery. Also, the prechemotherapy levels were significantly lower than the post-surgery levels (Fig. 4).

The circulating miR-let 7 levels were significantly higher in the pre-surgery and post-chemotherapy BC patients when compared to the control group. In the surgery group of patients, the post-surgery levels of miR-let 7 were significantly lower when compared with the pre-surgery levels. In contrast, in the chemotherapy group of patients, the post-chemotherapy levels of miR-let 7 were significantly higher than the prechemotherapy levels (Fig. 5).

The index (let 7/H19) was calculated by dividing the let 7 levels by the corresponding $\mathrm{H} 19$ levels in each subject, hence combining the changes of two circulating markers, the alleged tumor suppressor miR-let-7 and the oncogenic IncRNA H19. The ratio was significantly lower in the pre-surgery BC patients compared to the control group. The ratio then showed subsequent stepwise increase in the post-surgery, prechemotherapy and post-chemotherapy patients. The highest ratio observed in the post-chemotherapy $\mathrm{BC}$ patients, showing an almost 7-fold increase when compared to the control group. The ratio was significantly increased in the prechemotherapy group when compared with the post-surgery group. The level significantly increased in the post-chemotherapy group when compared with the prechemotherapy group (Fig. 6).

\subsection{Correlation studies}

A moderate inverse correlation was found between the circulating miR-let 7 levels versus CA $15-3$ in the pre-surgery study group (Fig. 7).

There is a direct strong correlation between $\mathrm{H} 19$ and total miR-675-5p levels (Fig. 8) and a direct moderate correlation between $\mathrm{H} 19$ and miR-675-3p (Fig. 9).

\subsection{Relation Studies}

Evaluation of the association of clinicopathological parameters in relation to the circulating ncRNAs measured in the present study showed a significantly increased level of miR-675-3p in patients with a positive family history versus those with no family history of BC (Fig. 10). Also, the circulating level of miR-let 7 was significantly associated with tumor size; a significantly lower level in tumor sizes of more than $2 \mathrm{~cm}$ when compared to tumors of size less than $2 \mathrm{~cm}$ (Fig. 11).

No statistically significant association was found between the clinicopathological parameters and the circulating levels of ncRNAs in the prechemotherapy BC patients (Fig. 12, Fig. 13 and Fig. 14).

\subsection{Validity Studies}


Validity analysis studies to discriminate pre-surgery patients from control revealed the highest AUC for miR-675-3p, with an AUC of 1.00 (95\%: $0.957-1.012) \mathrm{Cl}$, a cut off value of 2.53 having a $100 \%$ sensitivity, specificity, positive predictive value (PPV) and negative predictive value (NPV). CA $15-3$ has AUC of 0.984 with a cut of value $>13.1 \mathrm{U} / \mathrm{ml}$, with a sensitivity of $96.7 \%$ and specificity of $93.3 \%$. LncRNA H19 had an AUC of 0.956 with a cutoff value of $>1.65$. MiR-675-5p has an AUC of 0.951 with a cut off value of $>1.45$, with a sensitivity of $90 \%$ and specificity of $83.3 \%$. Let $7 / \mathrm{H} 19$ ratio showed an AUC of 0.749 with a cut off value of $\leq 0.51$, and sensitivity of $83.3 \%$ and specificity of $80 \%$ (Fig. 15). A combination of CA $15-3$ with IncRNA H19 yielded an AUC of 1.00 with sensitivity, specificity, PPV and NPV of $100 \%$ (Fig. 16).

\section{Discussion}

In the present study, CA 15 - 3 was measured as a conventional circulating tumor marker in BC for comparison of the ncRNAs under investigation. CA $15-3$ serum levels were significantly higher in the two groups of BC patients; who underwent surgery only and who received adjuvant chemotherapy, compared with the control group. CA $15-3$ dropped significantly after surgery yet did not show a significant change after chemotherapy.

$\mathrm{H} 19$ is involved in many biological processes including apoptosis, cell proliferation, and invasion in several tumors including BC [11]. It is implicated in the development of BC and increases $G 1$ to $S$ cell cycle transition when up regulated [12]. High tumor expressions of $\mathrm{H} 19$ were associated with the size of the tumor, nodal status and hormone negativity, having a negative correlation with the prognosis of BC. Unfavorable disease free and overall survival were exhibited in patients with high H19 levels [13]. Moreover, another study has found that the overexpression of $\mathrm{H} 19$ brought about a reversal in the tumor inhibitory effects of Huaier extract, yet its knocking down sensitized BC cells to Huaier extract. This study promoted the idea that the $\mathrm{H} 19$ /miRNA-675/CBL pathway is responsible for the BC cell proliferation and induction of apoptosis [7]. In light of these findings, $\mathrm{H} 19$ serves as a potential biomarker for the diagnosis of $\mathrm{BC}$ as well as a useful tool in the assessment of the response to adjuvant and neoadjuvant chemotherapy in BC. Furthermore, the response to therapy was also correlated to $\mathrm{H} 19$, where overly expressed $\mathrm{H} 19$ increased $\mathrm{BC}$ cell drug resistance to doxorubicin [14]. The findings of the present study agree with the emerging evidence identifying $\mathrm{H} 19$ as an oncogene. Analysis of the circulating $\mathrm{H} 19$ in the present study revealed significantly higher levels in the pre-surgery BC patients when compared to the control group, the levels significantly dropped after surgery, yet were still significantly higher than the control group. A further significant drop in the BC patients about to receive adjuvant chemotherapy was measured with no significant change in circulating $\mathrm{H} 19$ upon receiving chemotherapy. Then, the circulating $\mathrm{H} 19$ level showed a further significant drop in the prechemotherapy group when compared to the postsurgery BC patients and the chemotherapy did not show further significant change in $\mathrm{H} 19$ levels.

With regards to the descendants of H19; miRNA 675-5p and 675-3p, literature review found that miRNA675 , whether alone or together with H19, plays a role in cellular invasion and migration [15]. A study of miRNA-675 in paraffin embedded tissues demonstrated that the expression of miRNA-675 was 
significantly higher in the tissues of patients with $\mathrm{BC}$ compared with the control group, where no association was found between expression levels and age, lymph node, stage and receptor status [16]. In agreement with this data, Matouk et al demonstrated that miRNA-675 indirectly targets slug leading to increase of cell invasion and in vivo metastasis [17]. Another study has also identified both $\mathrm{H} 19$ and miRNA-675 as key players in the activation of the invasion and migration of BC cells [15]. These findings contrast with the results of a study carried out on 63 non-metastasized BC patients in University Medical Center Hamburg-Eppendorf, where no deregulation of plasma miRNA- 675 was found in the entire BC group [16]. The discrepancy in the results of miRNA-675 in BC patients may be reasoned by the method of analysis and calculation of data. As there is no consensus concerning the data normalization in the circulation, miRNA-16-5p was chosen as a reference gene in the present study to normalize the IncRNA and microRNAs' data since miR-16-5p is one of the most frequently used reference gene according to literature [18]. While, in the study of Müller et al,[16] miR-484 and b-actin were used as references to normalize their miRNA and IncRNA data, respectively.

Findings of the present study showed an increase in the levels of miRNA descendants of H19; miR-675-5p and miR-675-3p, in the pre and post-surgery BC patients when compared to the control group. The postsurgery levels of miR-675-5p showed a significant decline compared with the pre-surgery level. In the BC patients receiving adjuvant chemotherapy, the prechemotherapy levels of miR-675-5p are not statistically different from the control levels, however, significantly lower than the post-surgery group's levels. The circulating levels of miR-675-3p (passenger strand of miR-675-5p) showed more prominent increase in BC patients compared to miR-675-5p. The highest circulating levels of miR-675-3p was observed in the presurgery patients while the lowest levels were detected in the post-chemotherapy patients. The postsurgery levels were significantly lower than the pre-surgery. Moreover, the prechemotherapy levels were significantly lower than the post-surgery levels.

Literature review of miR-let 7 in $\mathrm{BC}$ has been controversial. On one hand, let-7a is well characterized as a tumor suppressor, with downregulated levels in several solid organ cancers [19]. It has also been linked to chemosensitivity of cancer in vitro cell lines, where miR-let 7 has been found to be an important predictor for the clinical response to the anthracycline drug-epirubicin, hence has been suggested to be a therapeutic target to regulate resistance from epirubicin-based chemotherapy [20]. Research findings indicate that lower expression of let-7a miRNA can induce chemoresistance in breast cancer by enhancing cellular apoptosis and suggest that let-7a may be used as a therapeutic target to modulate epirubicin-based chemotherapy resistance [20]. This phenomenon, although proven in cell lines, has not yet been proven in clinical breast tumors. On the other hand, miR-let 7 levels were unexpectedly almost 11.2 fold increased in BC patients when compared with the control group. After tumor resection, levels significantly dropped to levels comparable to that of the control group [21]. These findings were in line with the results of the present study which show significantly increased levels of circulating miR-let 7 in the pre-surgery and post-chemotherapy $\mathrm{BC}$ patients when compared to the control group. Although initially high in the $\mathrm{BC}$ patients, surgery brought upon a significant reduction. In contrast, chemotherapy brought about a significant increase in the levels of miR-let 7 in the $\mathrm{BC}$ group receiving adjuvant chemotherapy. 
The finding that miR-let 7 was greatly increased in the blood of $\mathrm{BC}$ patients raises an interesting question concerning the origin of the circulating miRNAs. The mechanism of delivery of tumor associated ncRNAs into the bloodstream remains unknown. Two hypotheses were raised by Slack et al in a report. [22] Primarily, tumor cell lyses and death releases ncRNAs into the circulation. A second hypothesis is that tumor cells expel ncRNAs across new blood vessels and pour into the circulation of the microenvironment of the tumor[22]. The upregulation after chemotherapy could indicate that the patients in this study showed hypothetically good response to chemotherapy and could possess a good prognosis for their treatment, yet the upregulation in the non-intervened $\mathrm{BC}$ patients could be as a feedback mechanism for the cells in response to the elevated $\mathrm{H} 19$ levels.

From the assumption above, a consequent ratio was derived from the present work of miR-let 7 to $\mathrm{H} 19$ demonstrating a theoretical tumor suppressor to oncogene level analysis. Statistical analysis of this ratio revealed that the pre-surgery group had a significantly lower ratio when compared with the control group. This ratio increased significantly in the prechemotherapy group when compared to the post-surgery group and showed a further statistically significant increased after receiving chemotherapy, becoming also significantly higher than the control group in the post-chemotherapy group.

The results of the present study, in terms of the effect of surgery on the levels of the ncRNAs, revealed a significant decrease in the levels of CA $15-3, \mathrm{H} 19$, miR-675-5p, miR-675-3p and miR-let 7. The ratio of miR-let 7 to $\mathrm{H} 19$ showed no statistical difference after surgery. Chemotherapy on the other hand, showed no statistically significant difference between the prechemotherapy and post-chemotherapy levels of $\mathrm{H} 19$, miR-675-5p and miR-675-3p. MiR-let 7 levels as well as let 7 to H19 ratio showed a statistically significant rise in the post-chemotherapy group when compared with the prechemotherapy group.

Correlation studies from the present work identified a direct strong correlation between $\mathrm{H} 19$ and miR-675$5 p(r=0.753, p<0.001)$ and a direct moderate correlation between H19 and miR-675-3p $(r=0.448, p<$ $0.05)$. This could be explained as an increase in precursor $\mathrm{H} 19$ results in a consequent increase in its descendants miR-675-5p and - 3p. Although this finding agrees with most of the studies assessing the H19/ miR-675 in BC patients, a striking finding was found where opposite levels were reported with an increase in $\mathrm{H} 19$ accompanied by a decrease in miRNA-675 in the HER2-positive BC subgroup [16]. No correlation was found between $\mathrm{H} 19$ and let7 levels in the $\mathrm{BC}$ in the present study, yet an inverse moderate correlation between CA $15-3$ and miR-let 7 level was found $(r=-.0364, p<0.05)$. This goes in alignment with literature identifying CA $15-3$ as a tumor invasion promoter and miR-let 7 as a tumor suppressor.

Relation studies were evaluated in the pre-surgery group assessing the effect of family history, menopause, grade, lymph invasion, tumor size and stage. No relation was found in the levels of the ncRNAs in the present study with regards to the menopausal state, grade and stage. No relation was also found in nodal status subgroups, in agreement with a previous study performed on BC tissue of patients finding no differences between nodal status subgroups [16]. The results revealed a significantly increased miR-675-3p level in patients with a positive family history of breast cancer $(p<0.001)$. From this finding we could derive a non-invasive method for the surveillance of circulating miR-675-3p in individuals with a 
positive family history of BC. An inverse relation was also identified from the current study between tumor size and miR-let 7, where a significantly lower level in tumor sizes of more than $2 \mathrm{~cm}$ as compared with tumors of less than $2 \mathrm{~cm} .(p<0.001)$ This could potentially provide a more accurate estimate to tumor size before surgery, being of substantial value in neoadjuvant therapy, where tumor size could be monitored easily and directly using circulating miR-let 7 levels.

Among the chemotherapy group, relation studies relating receptor status; ER, PR and HER2 were analyzed together with their intrinsic subtyping classification and the triple negativity. Although no statistically significant results were concluded, miR-675-3p levels were notably higher in the HER2 positive group when compared with the negative group with a significance of 0.077 . This shows similar findings to the study by Müller et al[16], where an association was found between circulating H19 and HER2 overexpression [16]. They were also remarkably higher in the luminal B group when compared to the luminal A group. These difference although have not proven significance in the present study, indicate that levels of miR-675-3p are higher in subgroups with worse prognosis. More conclusive results could be attained by performing the study on a larger sample size of BC patients.

Validity analysis of the parameters measured in this study displayed a maximal AUC for miR-675-3p at a cut off value of $>2.53$, with an AUC of 1.000 and $100 \%$ sensitivity, specificity, PPV and NPV. CA $15-3$ produced an AUC of 0.984 (95\% Cl: $0.957-1.012$ ) with a cut of value $>13.1 \mathrm{U} / \mathrm{ml}$, having a sensitivity and specificity of $96.7 \%$ and $93.3 \%$ respectively. Although exhibiting an excellent sensitivity and specificity in this study, a cut off value of $13.1 \mathrm{U} / \mathrm{ml}$ is not a reflection of the acceptable reference level used by the laboratory standards. Analysis of H19 shortly followed with an AUC of 0.956 (95\% Cl: $0.895-1.016)$ with a cutoff value of $>1.65$ and a sensitivity and specificity of 90.0 and 86.67 respectively, making it a very strong diagnostic tool for BC. MiR-675-5p produced an AUC of 0.951 (95\% $\mathrm{Cl} 0.893-1.009)$ with a cut off value of $>1.45$, a sensitivity of $90 \%$ and specificity of $83.3 \%$. Let 7 exhibited an AUC of $0.941(95 \% \mathrm{Cl}$ : $0.887-0.995$ ) at a cut off value $>1.32$ with a sensitivity and specificity of $96.67 \%$ and $80 \%$ respectively. The let 7/ H19 index yielded a weaker diagnostic power with an AUC of 0.749 (95\% Cl: 0.613-0.885) with a cut off value of $\leq 0.51$, sensitivity of $83.3 \%$ and specificity of $80 \%$. The combination of the conventional CA 15 - 3 with the $\mathrm{H} 19$ in the present study further enhanced the discriminative power of this test, produce a viable complement to current BC detection strategies yielding an AUC of 1.000 with $100 \%$ sensitivity, specificity, PPV and NPV.

In conclusion, the panel of ncRNAs in this study can all potentially act as novel biomarkers whether alone or combined in the diagnosis of BC. Surgery results in a significant drop in the levels of all H19, miR-675$5 p$, miR-675-3p and miR-let 7. Adjuvant chemotherapy did not result in a profound effect on the levels of ncRNAs in this study, yet it resulted in a statistically significant increase in the level of miR-let 7. Significantly increased miR-675-3p was associated with a positive family history of BC, making it a potential tool for assessing the onset of disease in females with a family history of BC. Elevated miR-let 7 levels were associated with smaller tumor sizes, if validated, could provide a non-invasive method for tumor size surveillance. 
The present study was not free from limitations. Results could be further validated and verified by adopting a larger sample size to obtain a more representative significance that is true to value, measuring the levels of ncRNAs in another group of subjects with benign tumors, following through with the same non-intervened $\mathrm{BC}$ patients collecting blood before and after surgery as well as before and after chemotherapy for the same patients and assessing the levels of the studied panel of ncRNAs in patients receiving neoadjuvant chemotherapy followed by surgery.

\section{Conclusions}

From the present study several findings could be concluded. The panel of ncRNAs in this study can all potentially act as novel biomarkers whether alone or combined in the diagnosis of BC. Surgery results in a significant drop in the levels of all H19, miR-675-5p, miR-675-3p and miR-let 7. On the other hand, adjuvant chemotherapy did not result in a profound effect on the levels of ncRNAs in this study, yet it resulted in a statistically significant increase in the level of miR-let 7 . Furthermore, significantly increased miR-675-3p was associated with a positive family history of $B C$, making it a potential tool for assessing the onset of disease in females with a family history of BC. Moreover, elevated miR-let 7 levels were associated with smaller tumor sizes, if validated, could provide a non-invasive method for tumor size surveillance.

\section{Declarations}

Acknowledgments: I would like to thank the nursing teams of the Surgery and the Cancer Management and Research departments of the Medical Research Institute for their cooperation and guidance during sample collection.

Author Contributions: Conceptualization, Nadine Wehida; methodology, Maher A. Kamel, Wafaa AbdelRehim and Nadine Wehida; validation and formal analysis, Maher A. Kamel, writing original draft preparation, Nadine Wehida; writing- reviewing and editing, Wafaa Abdel-Rehim, Maher A. Kamel and Nadine Wehida, surgery patients recruitment, Ahmed Karmouty; chemotherapy patients' recruitment and resources, Hazem El Mansy; supervision, Wafaa Abdel-Rehim, Maher A. Kamel and Hazem El Mansy; funding acquisition, Nadine Wehida.

All authors have read and agreed to the published version of the manuscript.

Funding: This research received no external funding.

Institutional Review Board Statement: The study was conducted according to the International Conference on Harmonisation- Good Clinical Practice (ICH GCP) guidelines, and approved by the Ethics Committee of the Medical Research Institute, Alexandria University (Date of approval: 12/9/2019, approval serial number: E/C. S/N. T4/2019).

Data Availability Statement: Data supporting reported results can be provided upon request. 
Competing Interests: There are no financial or non-financial conflicts of interests to disclose.

Ethics Approval: This study was approved by the Ethics Committee of the Medical Research Institute, Alexandria University (Date of approval: 12/9/2019, approval serial number: E/C. S/N. T4/2019).

Consent to Participate: Informed consent was obtained from all subjects involved in the study.

Consent to Publish: Patient consent forms for publication purposes were obtained from all subjects involved in the study.

\section{References}

1. St Laurent G, Wahlestedt C, Kapranov P (2015) The Landscape of long noncoding RNA classification. Trends Genet 31:239-251. https://doi.org/ 10.1016/j.tig.2015.03.007

2. Ma L, Bajic VB, Zhang Z (2013) On the classification of long non-coding RNAs. RNA Biol 10:925933. https://doi.org/ 10.4161/rna.24604

3. Lee H, Han S, Kwon CS, Lee D (2016) Biogenesis and regulation of the let-7 miRNAs and their functional implications. Protein Cell 7:100-113. https://doi.org/ 10.1007/s13238-015-0212-y

4. Gao H, Hao G, Sun Y, Li L, Wang Y (2018) Long noncoding RNA H19 mediated the chemosensitivity of breast cancer cells via Wnt pathway and EMT process. Onco Targets Ther 11:8001-8012. https://doi.org/ 10.2147/ott.s172379

5. Vennin C, Spruyt N, Dahmani F, et al (2015) H19 non coding RNA-derived miR-675 enhances tumorigenesis and metastasis of breast cancer cells by downregulating c-Cbl and Cbl-b. Oncotarget 6:29209-29223. https://doi.org/ 10.18632/oncotarget.4976

6. Keniry A, Oxley D, Monnier P, Kyba M, Dandolo L, Smits G, Reik W (2012) The H19 lincRNA is a developmental reservoir of miR-675 that suppresses growth and Igf1r. Nat Cell Biol 14:659-665. https://doi.org/ 10.1038/ncb2521

7. Wang J, Wang X, Chen T, Jiang L, Yang Q (2017) Huaier Extract Inhibits Breast Cancer Progression Through a LncRNA-H19/MiR-675-5p Pathway. Cell Physiol Biochem 44:581-593. https://doi.org/ $10.1159 / 000485093$

8. Gilles ME, Slack FJ (2018) Let-7 microRNA as a potential therapeutic target with implications for immunotherapy. Expert Opin Ther Targets 22:929-939. https://doi.org/

10.1080/14728222.2018.1535594

9. Zhong G, Wang K, Li J, Xiao S, Wei W, Liu J (2020) Determination of Serum Exosomal H19 as a Noninvasive Biomarker for Breast Cancer Diagnosis. Onco Targets Ther 13:2563-2571. https://doi.org/ 10.2147/ott.s243601

10. Livak, K. J., \& Schmittgen, T. D. (2001). Analysis of relative gene expression data using real-time quantitative PCR and the 2- $\Delta \Delta C T$ method. Methods, 25(4), 402-408.

https://doi.org/10.1006/meth.2001.1262 
11. Qi P, Du X (2013) The long non-coding RNAs, a new cancer diagnostic and therapeutic gold mine. Mod Pathol 26:155-165. https://doi.org/ 10.1038/modpathol.2012.160

12. Berteaux N, Lottin S, Monté D, et al (2005) H19 mRNA-like noncoding RNA promotes breast cancer cell proliferation through positive control by E2F1. J Biol Chem 280:29625-29636. https://doi.org/ 10.1074/jbc.M504033200

13. Shima H, Kida K, Adachi S, et al (2018) Lnc RNA H19 is associated with poor prognosis in breast cancer patients and promotes cancer stemness. Breast Cancer Res Treat 170:507-516. https://doi.org/ 10.1007/s10549-018-4793-z

14. Wang X, Pei X, Guo G, Qian X, Dou D, Zhang Z, Xu X, Duan X (2020) Exosome-mediated transfer of long noncoding RNA H19 induces doxorubicin resistance in breast cancer. J Cell Physiol 235:68966904. https://doi.org/ 10.1002/jcp.29585

15. Peperstraete E, Lecerf C, Collette J, et al (2020) Enhancement of Breast Cancer Cell Aggressiveness by IncRNA H19 and its Mir-675 Derivative: Insight into Shared and Different Actions. Cancers (Basel) 12:1730. https://doi.org/ 10.3390/cancers 12071730

16. Müller V, Oliveira-Ferrer L, Steinbach B, Pantel K, Schwarzenbach H (2019) Interplay of IncRNA H19/miR-675 and IncRNA NEAT1/miR-204 in breast cancer. Mol Oncol 13:1137-1149. https://doi.org/ 10.1002/1878-0261.12472

17. Matouk IJ, Halle D, Raveh E, Gilon M, Sorin V, Hochberg A (2016) The role of the oncofetal H19 IncRNA in tumor metastasis: orchestrating the EMT-MET decision. Oncotarget 7:3748-3765. https://doi.org/ 10.18632/oncotarget.6387

18. Donati S, Ciuffi S, Brandi ML (2019) Human Circulating miRNAs Real-time qRT-PCR-based Analysis: An Overview of Endogenous Reference Genes Used for Data Normalization. Int J Mol Sci 20:4353. https://doi.org/ 10.3390/ijms20184353

19. Boyerinas B, Park SM, Hau A, Murmann AE, Peter ME (2010) The role of let-7 in cell differentiation and cancer. Endocr Relat Cancer 17:F19-36. https://doi.org/ 10.1677/erc-09-0184

20. Wu J, Li S, Jia W, Deng H, Chen K, Zhu L, Yu F, Su F (2015) Reduced Let-7a Is Associated with Chemoresistance in Primary Breast Cancer. PLoS One 10:e0133643. https://doi.org/ 10.1371/journal.pone. 0133643

21. Heneghan HM, Miller N, Lowery AJ, Sweeney KJ, Newell J, Kerin MJ (2010) Circulating microRNAs as novel minimally invasive biomarkers for breast cancer. Ann Surg 251:499-505. https://doi.org/ 10.1097/SLA.0b013e3181cc939f

22. Chin LJ, Slack FJ (2008) A truth serum for cancer-microRNAs have major potential as cancer biomarkers. Cell Res 18:983-984. https://doi.org/ 10.1038/cr.2008.290

\section{Figures}




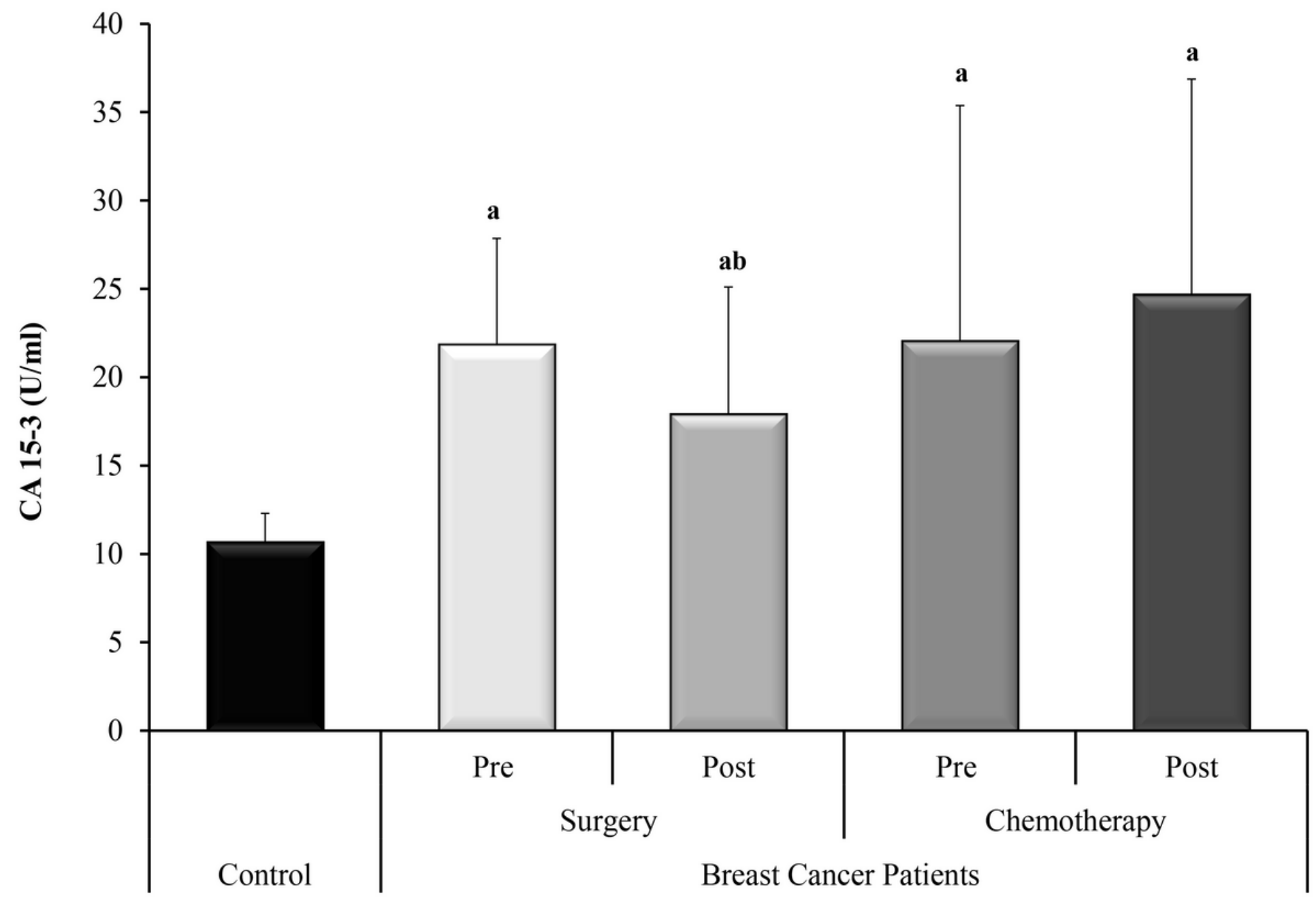

Figure 1

The levels of CA 15-3 in control subjects and BC groups; surgery and adjuvant chemotherapy a Significant between control and each group by using Post Hoc Test (Tukey) for ANOVA test b Significant between pre and post in same group by using Paired t-test 


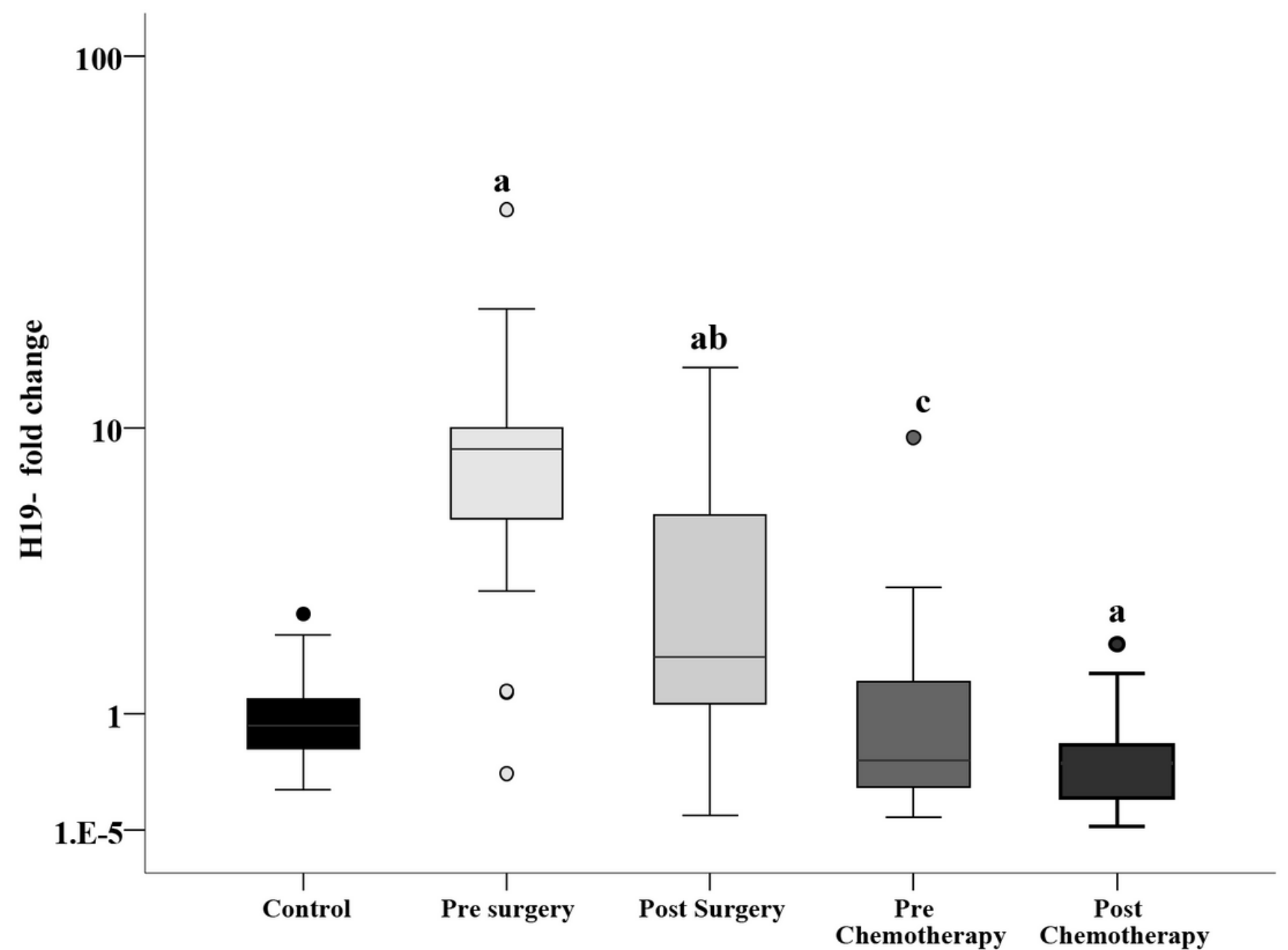

Figure 2

The circulating levels of $\mathrm{H} 19$ in the control subjects and the BC studied groups; surgery only and adjuvant chemotherapy

$1 . E-5=1 * 10^{-5}$

a Significant between control and each group by using Post Hoc Test (Dunn's), Kruskal Wallis test

b Significant between pre and post in same group by using Wilcoxon signed ranks test

c Significant between post-surgery and prechemotherapy by using Mann Whitney test

Figure 3

The circulating levels of miR-675-5p in the control subjects and the BC studied groups; surgery only and adjuvant chemotherapy 


\section{$1 . \mathrm{E}-5=1 * 10^{-5}$}

a Significant between control and each group by using Post Hoc Test (Dunn's), Kruskal Wallis test

b Significant between pre and post in same group by using Wilcoxon signed ranks test

c Significant between post-surgery and prechemotherapy by using Mann Whitney test

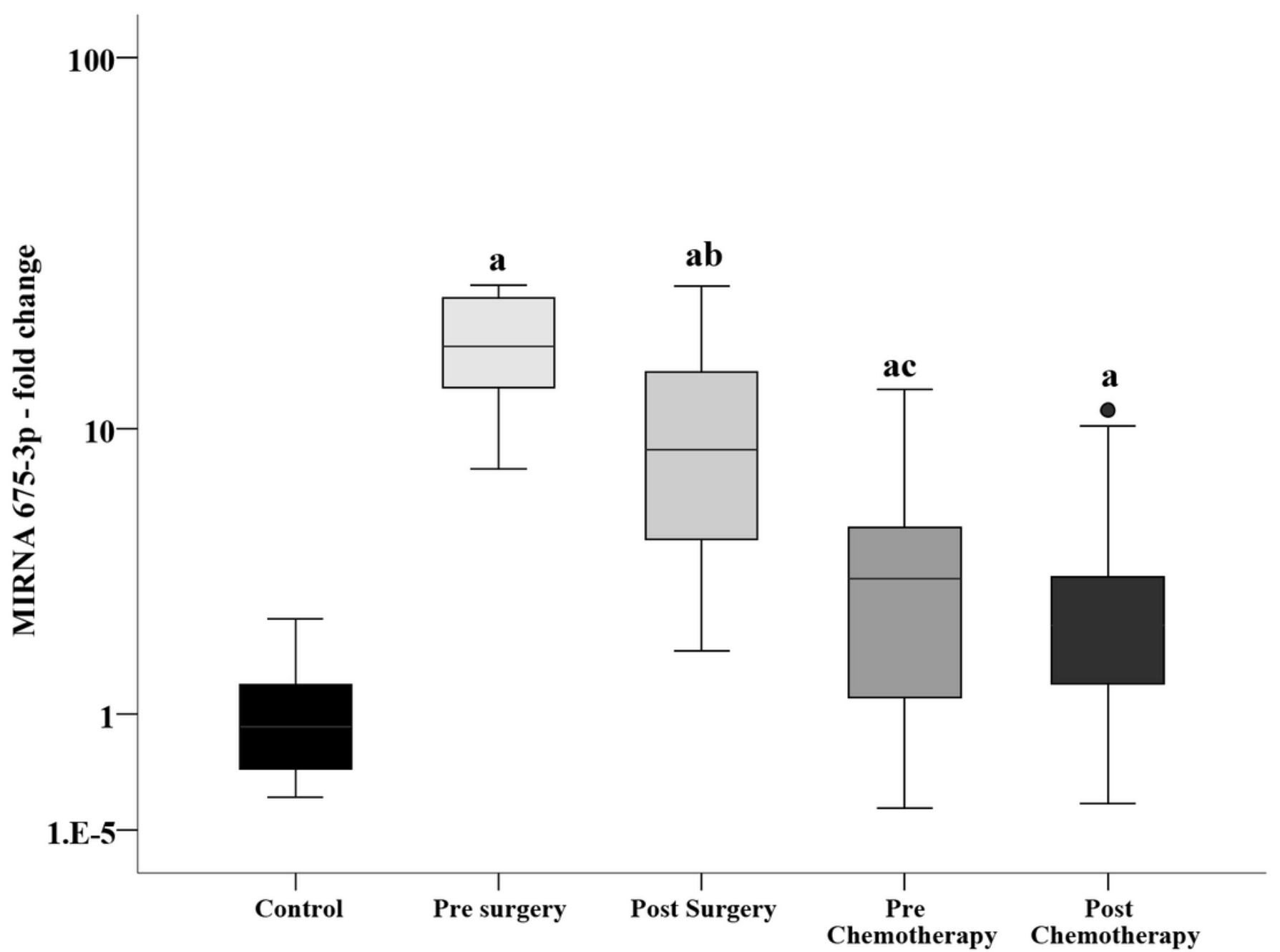

Figure 4

The circulating levels of miR-675-3p in the control subjects and the BC studied groups; surgery only and adjuvant chemotherapy

1. $E-5=1 * 10^{-5}$

a Significant between control and each group by using Post Hoc Test (Dunn's), Kruskal Wallis test

b Significant between pre and post in same group by using Wilcoxon signed ranks test

c Significant between post-surgery and prechemotherapy by using Mann Whitney test 


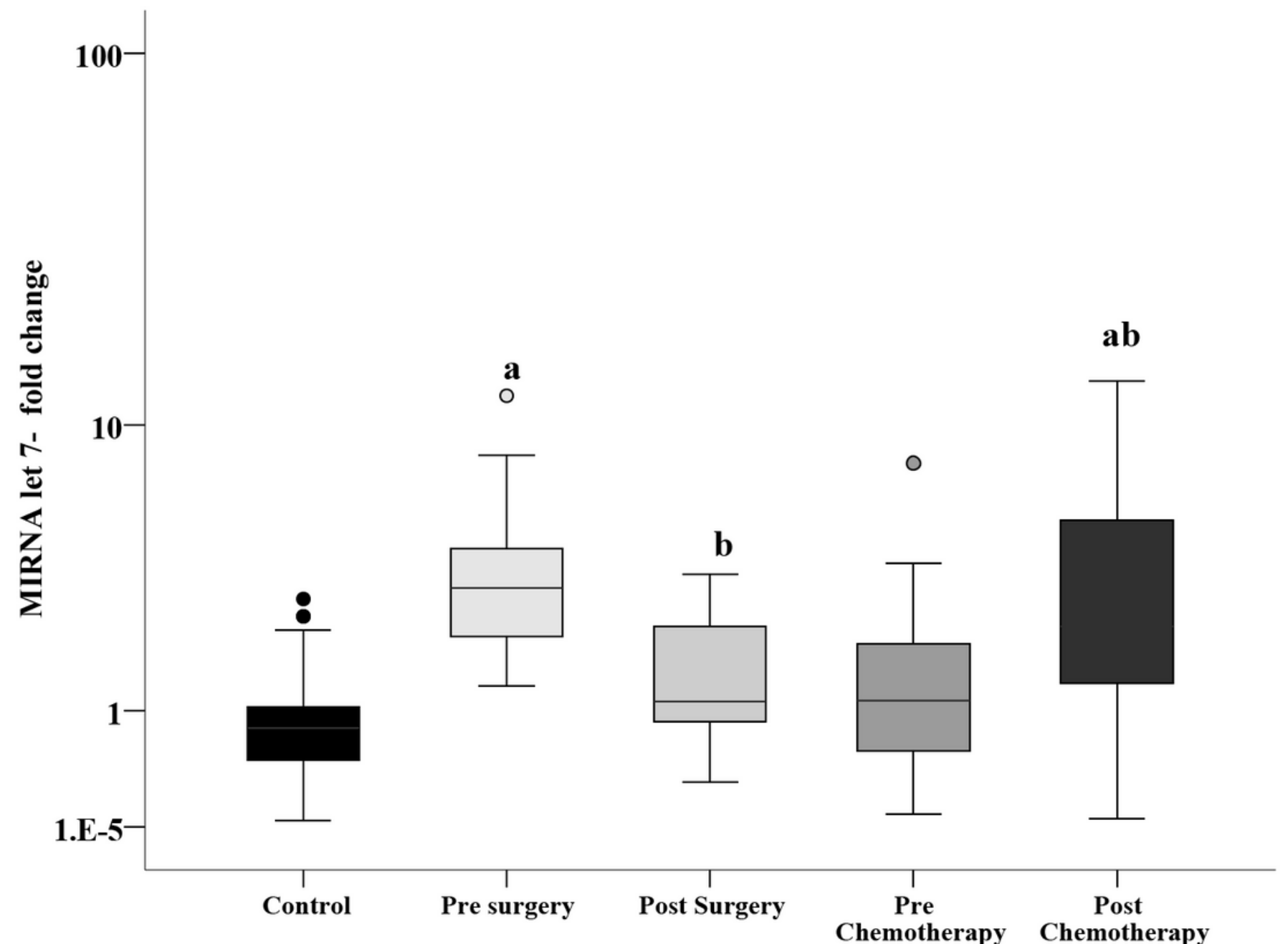

Figure 5

The circulating levels of miR-let 7 in the control subjects and the BC studied groups; surgery only and adjuvant chemotherapy

$1 . \mathrm{E}-5=1 * 10^{-5}$

a Significant between control and each group by using Post Hoc Test (Dunn's), Kruskal Wallis test

b Significant between pre and post in same group by using Wilcoxon signed ranks test 


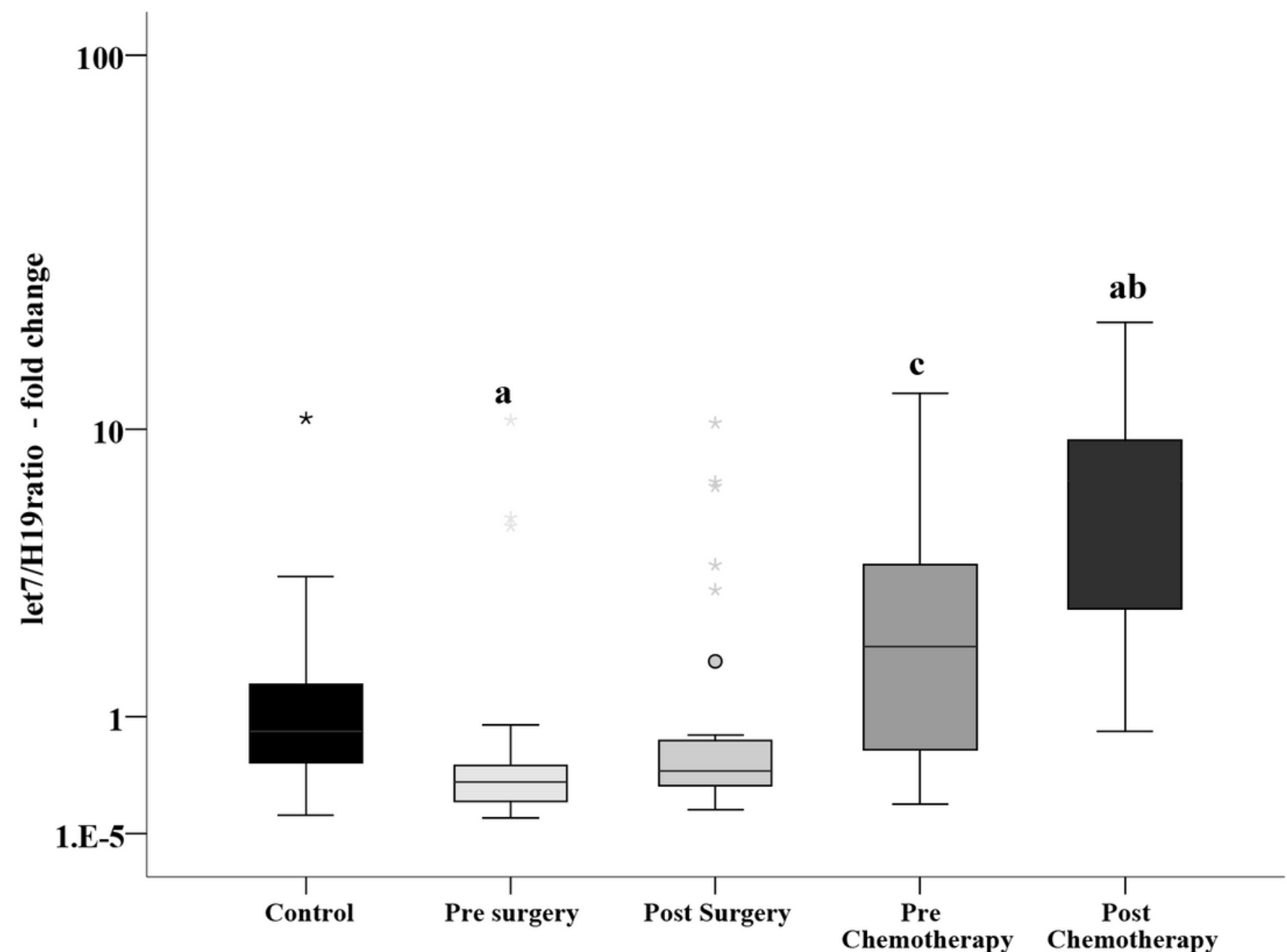

Figure 6

The let $7 / \mathrm{H} 19$ ratio in the control subjects and the $\mathrm{BC}$ studied groups; surgery only and adjuvant chemotherapy

$1 . \mathrm{E}-5=1 * 10^{-5}$

a Significant between control and each group by using Post Hoc Test (Dunn's), Kruskal Wallis test

b Significant between pre and post in same group by using Wilcoxon signed ranks test

c Significant between post-surgery and prechemotherapy by using Mann Whitney test 


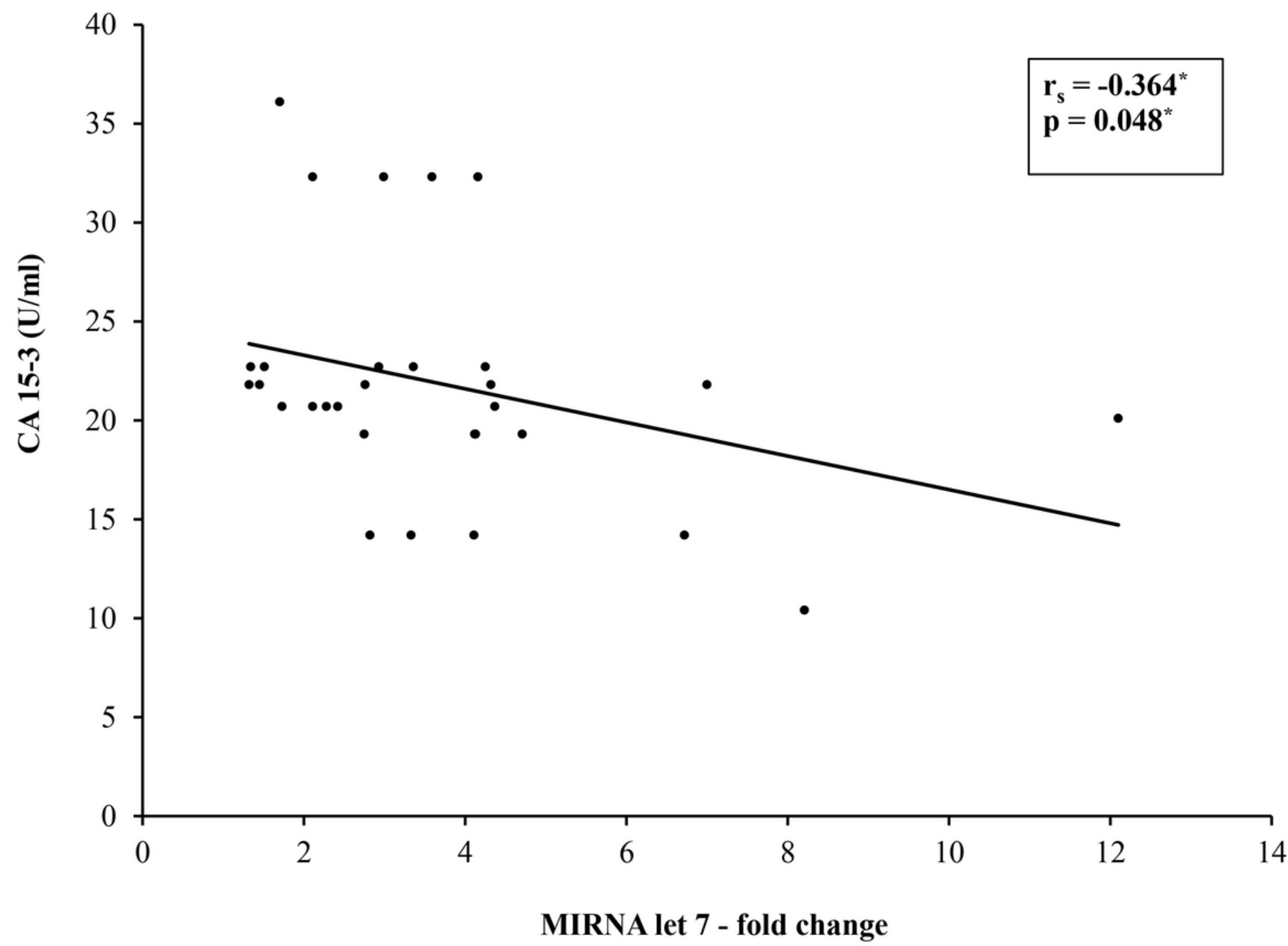

Figure 7

Correlation curve between CA 15-3 and circulating miR-let 7 in pre-surgery BC patients $(n=30)$ 


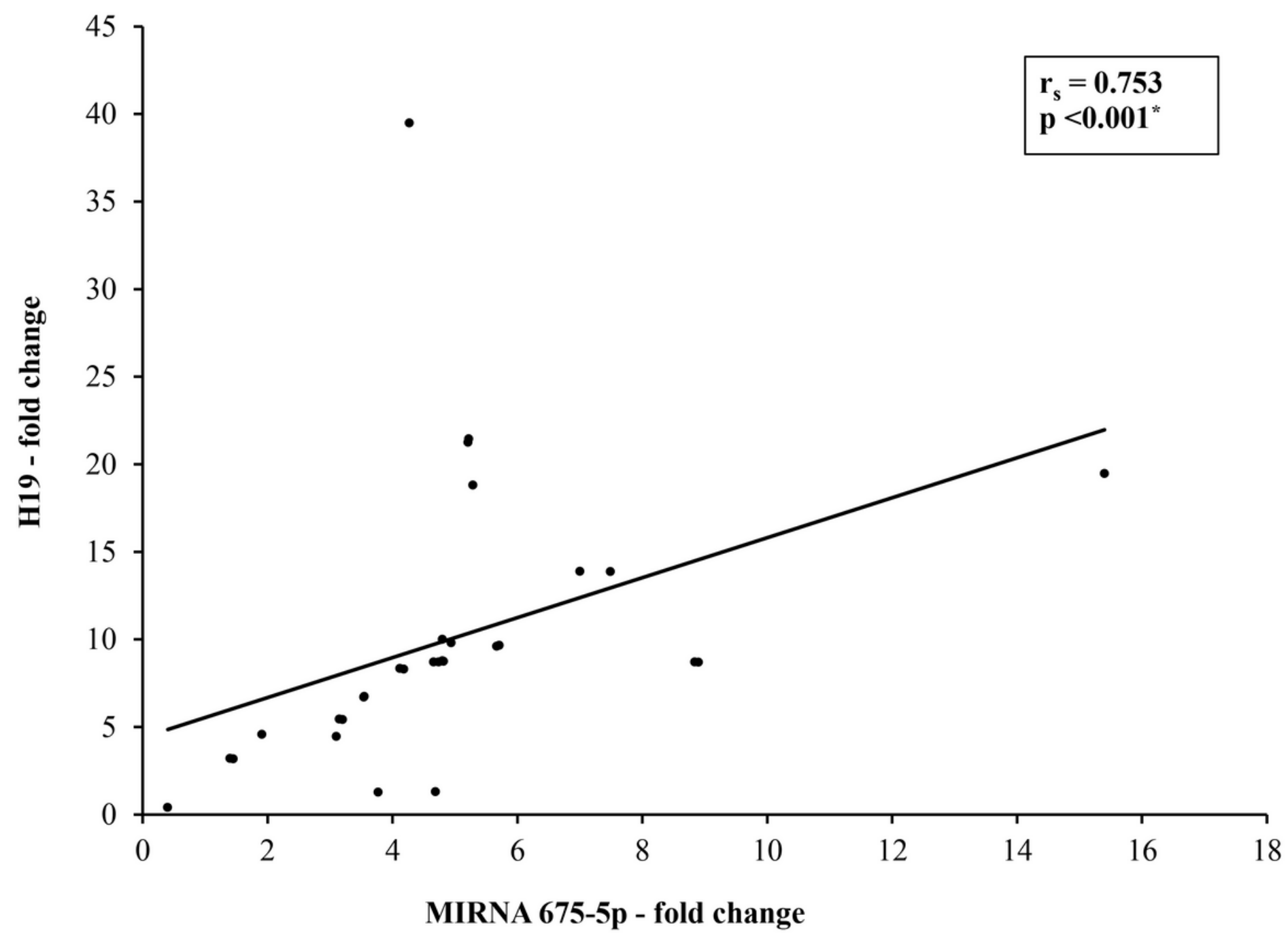

Figure 8

Correlation between circulating $\mathrm{H} 19$ with miR-675-5p in pre-surgery $\mathrm{BC}$ patients 


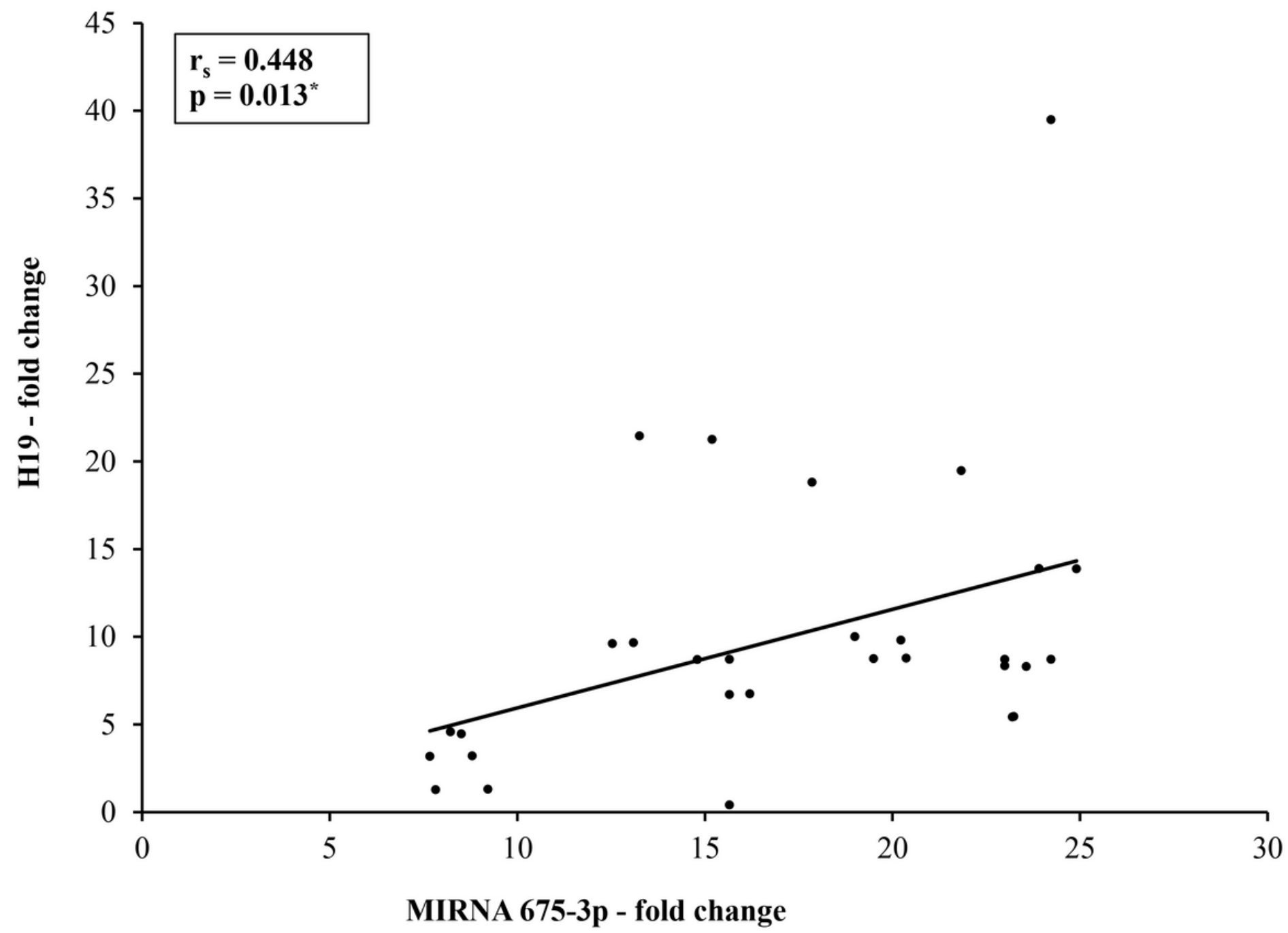

Figure 9

Correlation between circulating $\mathrm{H} 19$ with miR-675-3p in pre-surgery $\mathrm{BC}$

*: Statistically significant at $p \leq 0.05$ 


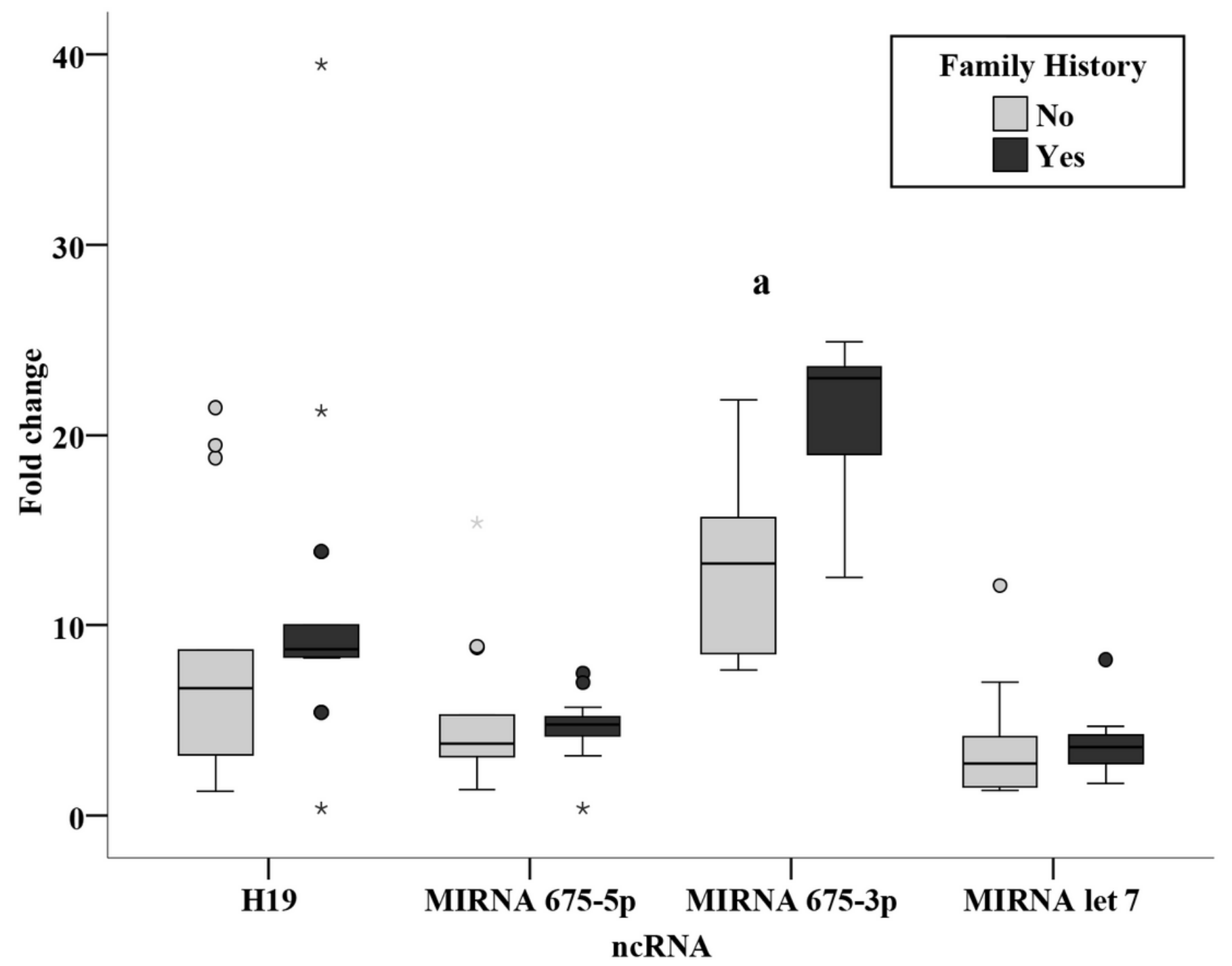

Figure 10

Relation between ncRNAs and family history in pre-surgery BC patients

a: Significant between ncRNA and family history using Mann Whitney test 


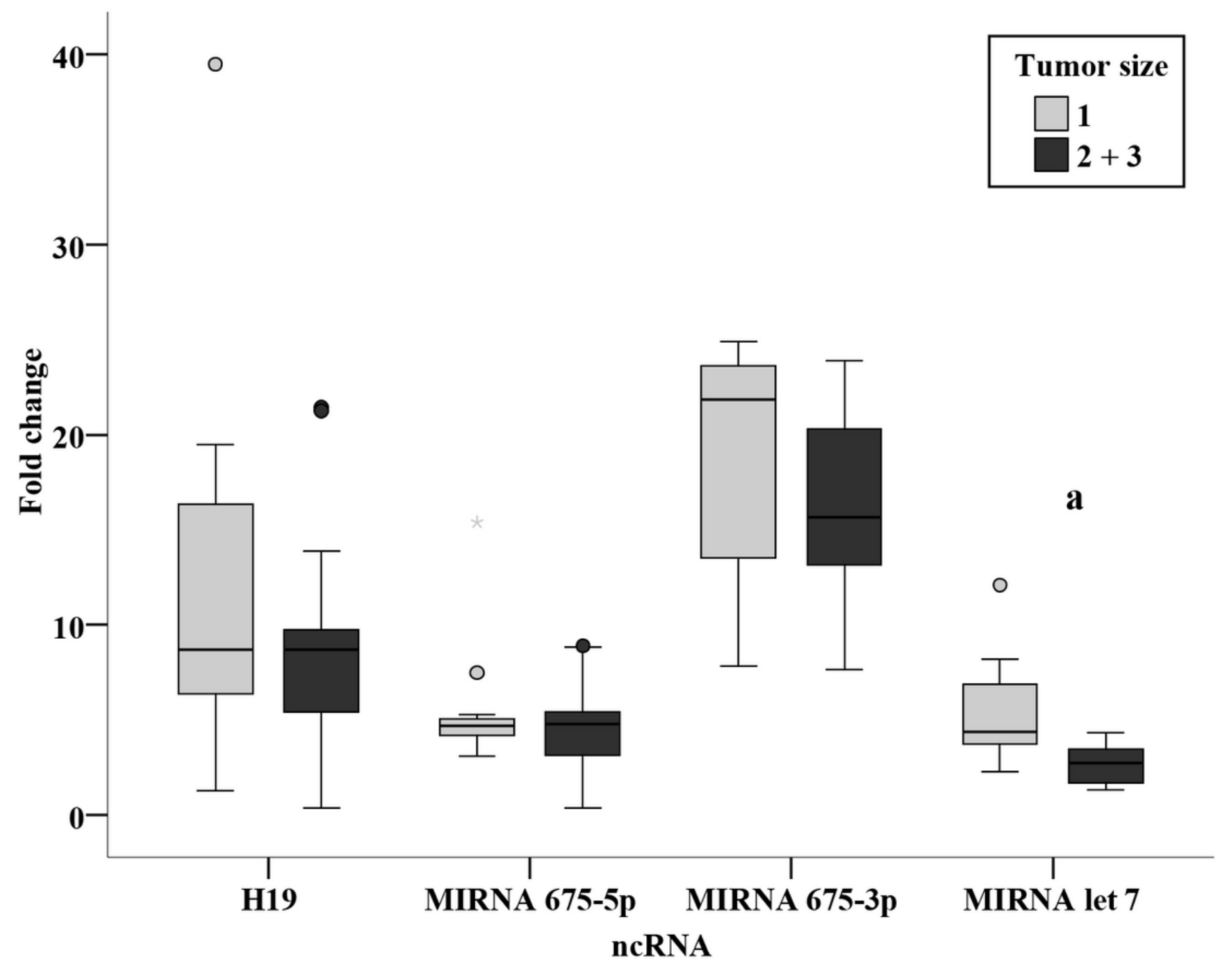

Figure 11

Relation between ncRNA and tumor size in pre-surgery $\mathrm{BC}$ patients

a: Significant between ncRNA and tumor size using Mann Whitney test 


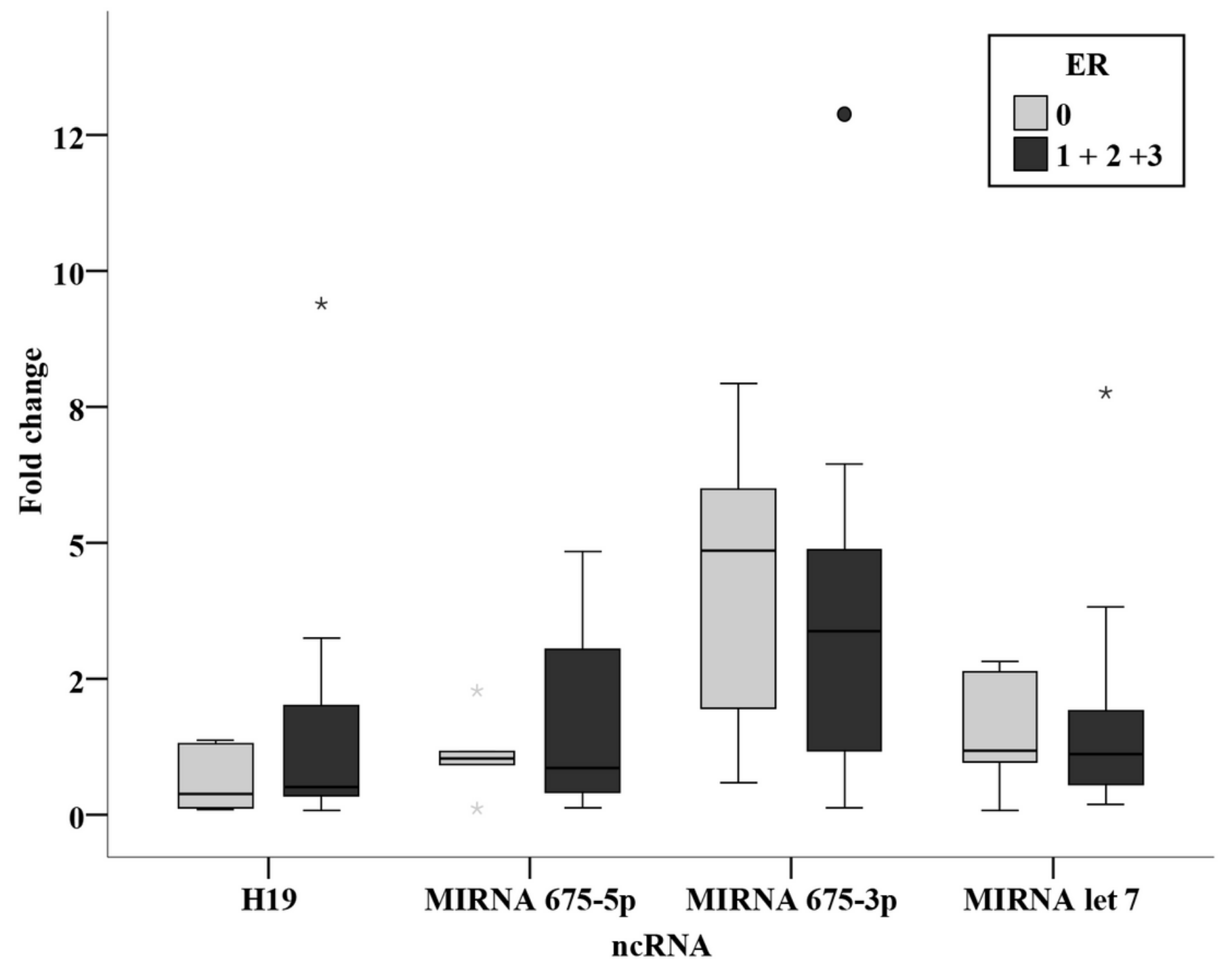

Figure 12

Relation between ncRNAs and ER status in the prechemotherapy BC patients 


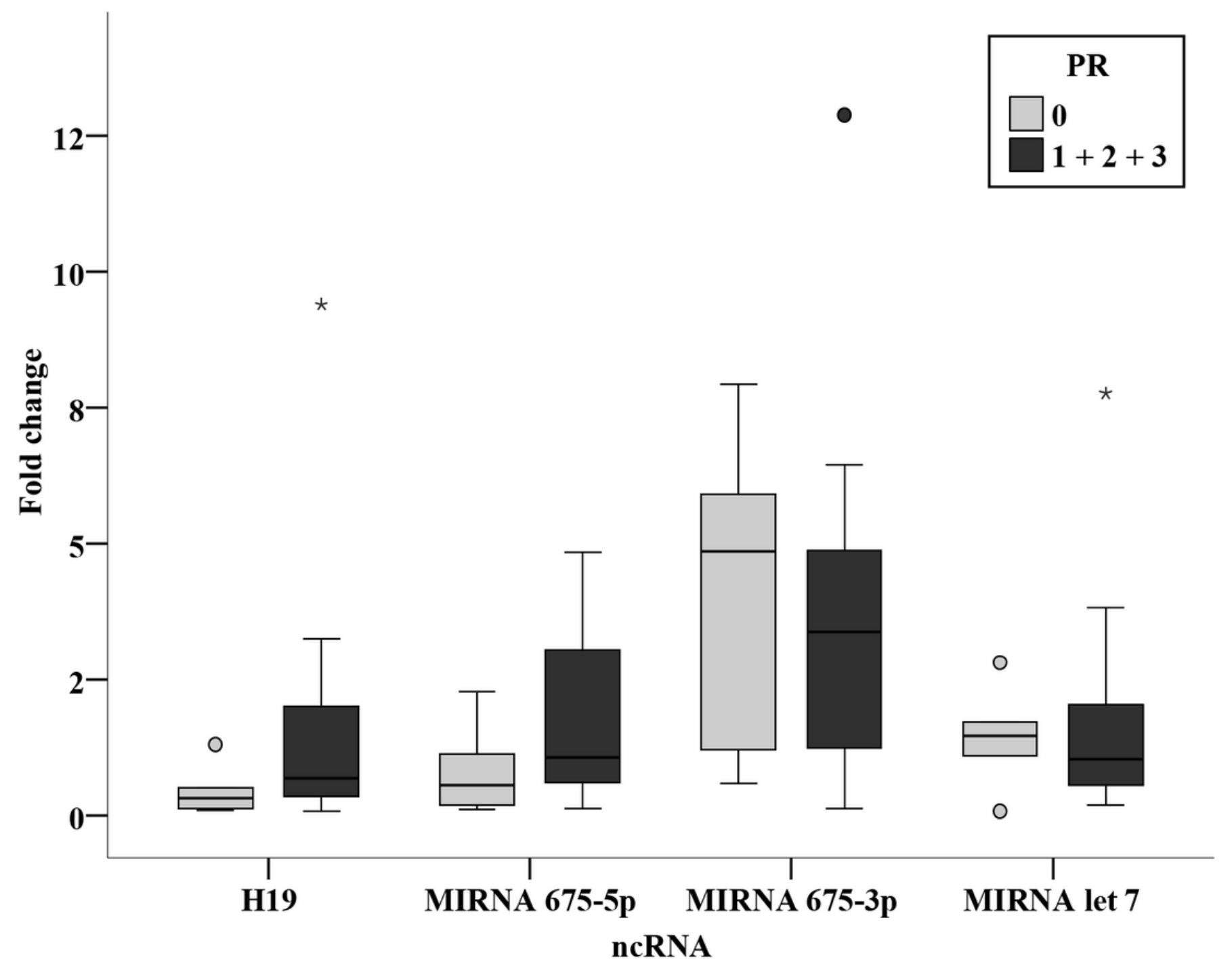

Figure 13

Relation between ncRNAs and PR status in the prechemotherapy BC patients 


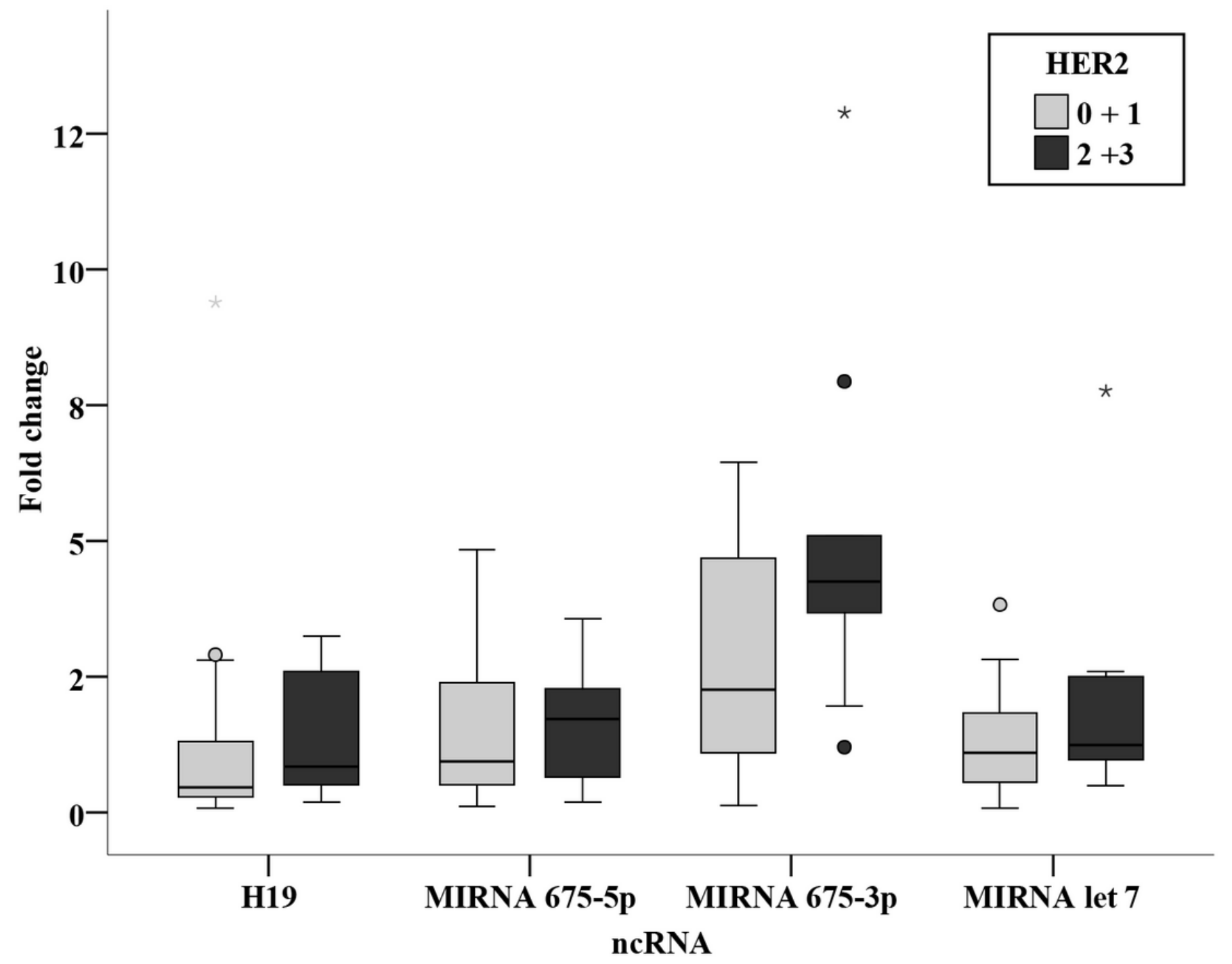

Figure 14

Relation between ncRNAs and HER2 status in the prechemotherapy BC patients 


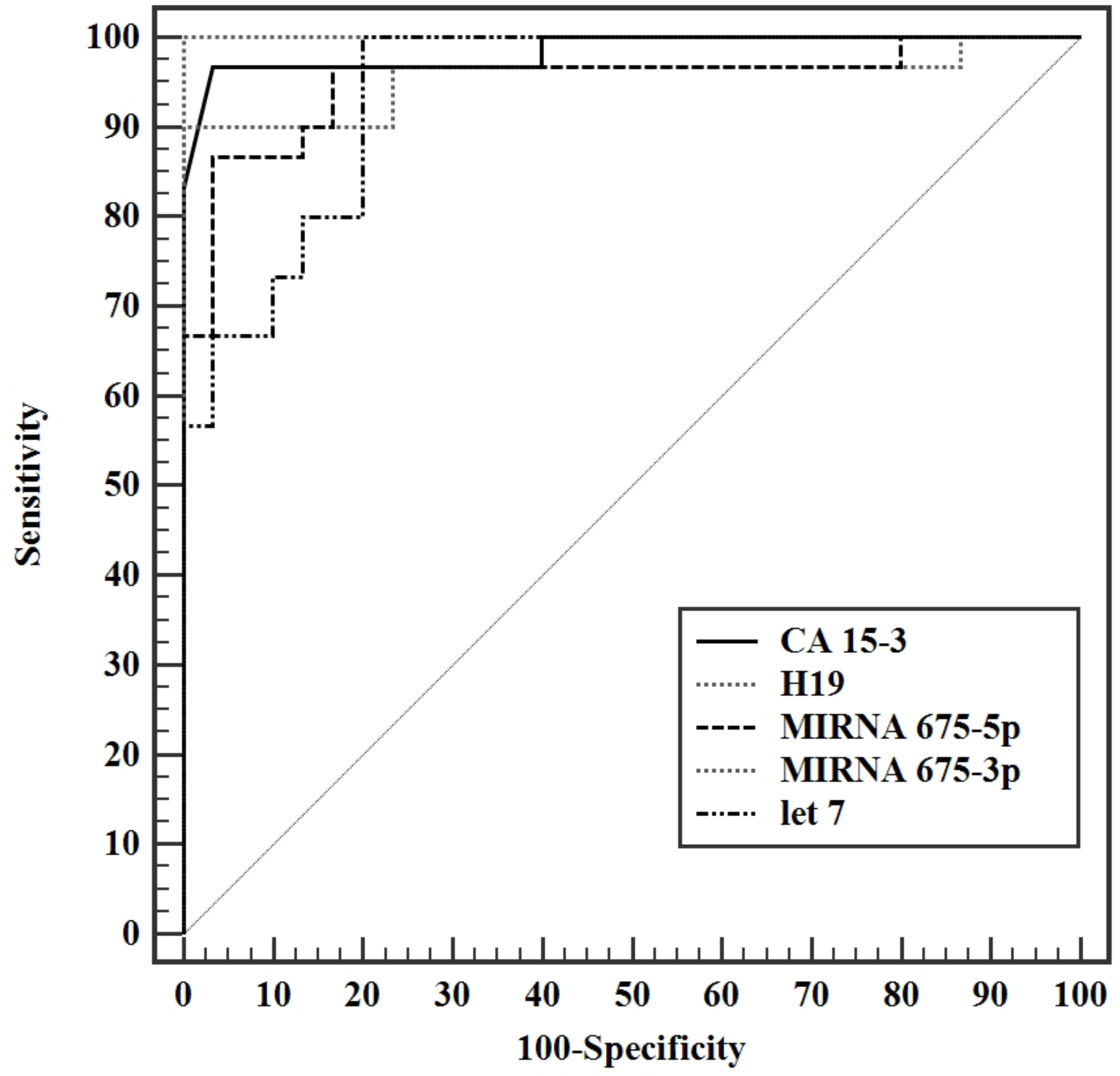

Figure 15

ROC curve for CA 15-3 (U/ml), H19, MIRNA 675-5p, MIRNA 675-3p and let 7 to discriminate pre surgery patients $(n=30)$ from control $(n=30)$ 


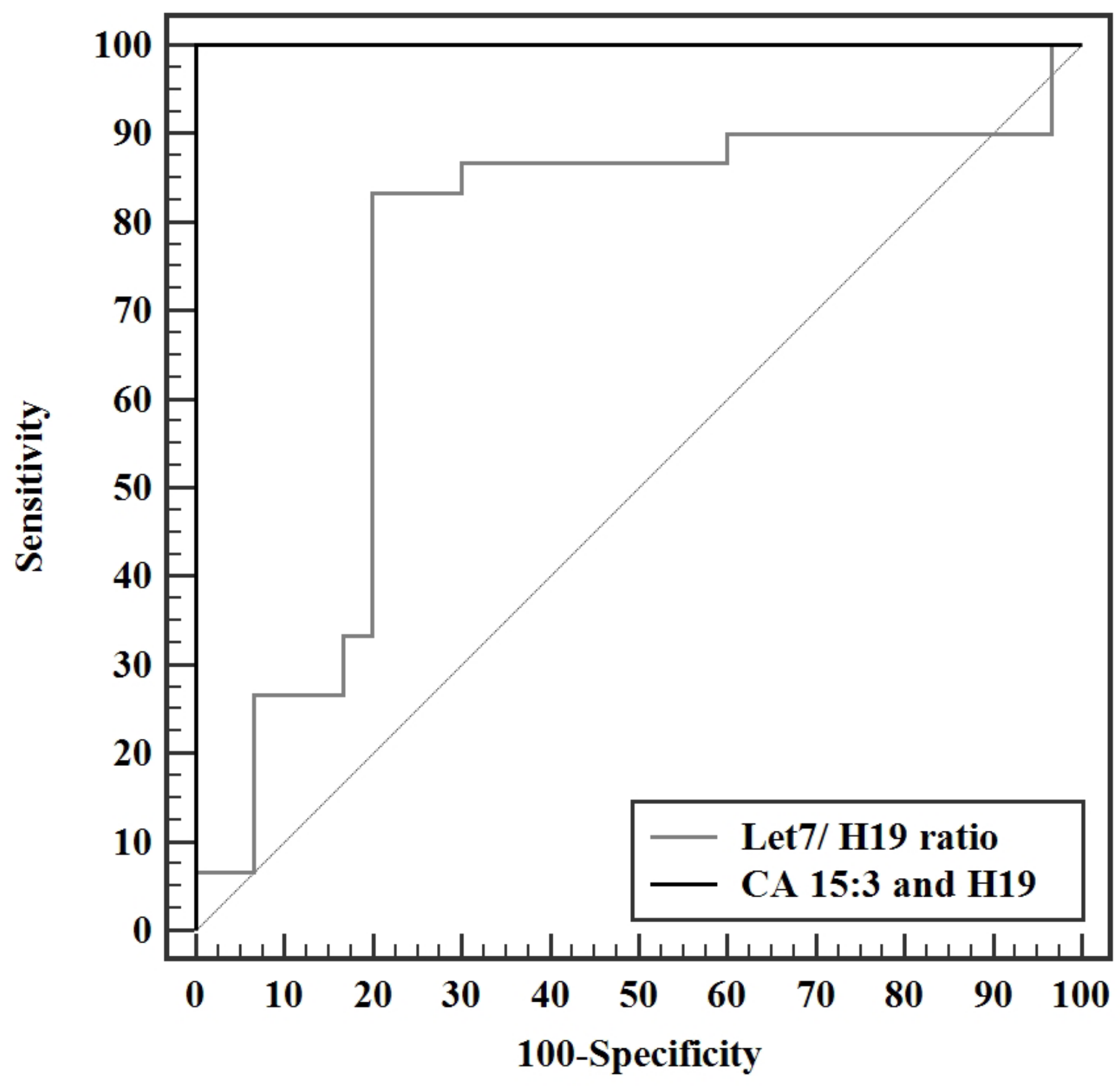

Figure 16

ROC curve for let7/H19 ratio and the combination of CA $15: 3$ and $\mathrm{H} 19$ to discriminate pre surgery patients $(n=30)$ from control $(n=30)$ 